Bundesgesundheitsbl 2016 · 59:1587-1592 DOI 10.1007/s00103-016-2459-8

Online publiziert: 24. Oktober 2016

(c) Der/die Autor(en) 2016. Dieser Artikel ist eine Open-Access-Publikation.

CrossMark
Claudia Riedel · Birka Lehmann · Karl Broich - Thomas Sudhop

Bundesinstitut für Arzneimittel und Medizinprodukte (BfArM), Bonn, Deutschland

\title{
Arzneimittelzulassung für Kinder und Jugendliche verbessern
}

\author{
Positionspapier zum Kinderarzneimittel- \\ Symposium am 8. Juni 2015 in Bonn
}

\section{Einleitung}

„Kinder sind keine kleinen Erwachsenen“. Dieser triviale, aber dennoch fundamentale Satz beschreibt die Diskussionen des Kinderarzneimittel-Symposiums des Bundesinstituts für Arzneimittel und Medizinprodukte (BfArM) am 8. Juni $2015 \mathrm{im}$ Wissenschaftszentrum Bonn.

150 Fachleute diskutierten über mögliche Maßnahmen zur Verbesserung der Arzneimittelversorgung von Kindern und Jugendlichen. Ziel dieses Symposiums war es, die Gründe für die geringe Zahl von Arzneimittelzulassungen für Kinder und Jugendliche im Bereich bekannter Wirkstoffe zu ergründen.

In acht Fachvorträgen referierten Vertreterinnen und Vertreter der Patientenorganisationen $^{1}$, des BfArMs, der Deutschen Gesellschaft für Kinder- und Jugendmedizin (DGKJ), der Verbände der pharmazeutischen Industrie und eines pharmazeutischen Unternehmens, des Gemeinsamen Bundesausschusses (G-BA) und des Deutschen Zentrums für Luft- und Raumfahrt (DLR) als Projektträger des Bundesministeriums für Bildung und Forschung (BMBF) über verschiedene Aspekte der Entwicklung von Arzneimitteln für Kinder und Jugendliche.

Erkenntnisse aus der Anwendung von Arzneimitteln für Erwachsene lassen sich im Allgemeinen nicht unmittelbar auf Kinder und Jugendliche übertragen. Viele zugelassene Arzneimittel werden auch heute noch bei Kindern und Jugendli-

\footnotetext{
${ }^{1}$ Deutsche Kinderkrebshilfe und Deutsche Leukämie-Forschungshilfe.
}

chen angewendet, ohne dass systematische klinische Studien zur Dosierung oder zur Darreichungsform für diese Altersgruppen vorliegen. Dabei scheint das Ausmaß des sogenannten Off-Label Use - also die Anwendung zugelassener Arzneimittel außerhalb ihrer Zulassungsbedingungen - bei Kindern und Jugendlichen rückläufig, aber dennoch insgesamt weiterhin beträchtlich zu sein [1-4].

Mit der Verordnung (EG) Nr. 1901/ 2006 über Kinderarzneimittel sollten die Entwicklung und die Zugänglichkeit von Arzneimitteln für die pädiatrische Bevölkerungsgruppe verbessert werden. Ziel der Verordnung war es, u. a. zu gewährleisten, dass die zur Behandlung der pädiatrischen Bevölkerungsgruppe verwendeten Arzneimittel im Rahmen ethisch vertretbarer und qualitativ hochwertiger Forschungsarbeiten entwickelt und auch eigens für die pädiatrische Verwendung zugelassen werden. Für neue sowie noch patentgeschützte Arzneimittel hat der europäische Gesetzgeber in der Verordnung eine Mischung verpflichtender Maßnahmen und Anreize geschaffen, die in den letzten Jahren insgesamt zu einer Verbesserung der Versorgungssituation von Kindern und Jugendlichen für diese Arzneimittel geführt haben.

Das ebenfalls in der Verordnung geschaffene, ausschließlich anreizbasierte Verfahren bei den sogenannten bekannten oder generischen („off-patent“) Arzneimitteln blieb allerdings bisher weitgehend erfolglos.

Dieses 2007 als „Paediatric Use Marketing Authorisation“ (kurz PUMAVerfahren) implementierte Zulassungsverfahren räumt den Inhabern dieser
Zulassungen eine 10-jährige Unterlagenschutzfrist für die beantragten pädiatrischen Indikationen ein, d.h. andere Hersteller dieser generischen Arzneimittel können auf diese Unterlagen innerhalb der ersten 10 Jahre nach Zulassung keinen Bezug nehmen und daher ohne eigene klinische Prüfungen keine Zulassungen für diese pädiatrischen Indikationen und Darreichungsformen erhalten. Als „Gegenleistung“ für diesen weitgehenden Unterlagenschutz muss der pharmazeutische Unternehmer jedoch ein pädiatrisches Prüfkonzept (Paediatric Investigation Plan, PIP) beim Pädiatrieausschuss der Europäischen Arzneimittelagentur vorlegen und genehmigen lassen und das Arzneimittel dann entsprechend der Entscheidung des Ausschusses entwickeln.

Im Rahmen des Symposiums wurden mögliche Gründe für die bisher sehr geringe Nutzung von PUMA-Zulassungen im Gegensatz zu den Maßnahmen und Anreizen für patentgeschützte Arzneimittel aus Sicht der verschiedenen Fachkreise diskutiert und sind nachfolgend zusammengefasst dargestellt.

\section{Situation aus Sicht der Pädiatrie und der Patientenorganisatio- nen}

Vertretende von Patientenorganisationen, Pädiatrie sowie des Projektträgers legten in ihren Vorträgen die Problematik der derzeitigen Versorgungssituation bei Kindern und Jugendlichen dar [5-8].

Während sich die Zulassungssituation für Arzneimittel mit neuen Wirkstoffen bei Kindern und Jugendlichen lang- 
sam verbessert, stellt sich die Situation für Generika weniger positiv dar. Aus Sicht der Patientinnen und Patienten sowie der behandelnden Ärzte und Ärztinnen besteht ein hoher therapeutischer Bedarf an generischen Arzneimitteln. Obwohl teilweise jahrzehntelang eingesetzt, fehlen z. B. validierte Dosisangaben für die verschiedenen Altersklassen. Daten aus der Studie zur Gesundheit von Kindern und Jugendlichen in Deutschland (KiGGS-Studie) legen nahe, dass trotz des langjährigen Gebrauchs dieser Arzneimittel Überdosierungen - aber auch wahrscheinlich viel häufiger - Unterdosierungen, besonders in den jungen $\mathrm{Al}$ tersgruppen, vorkommen [4]. Die immer noch viel geübte Praxis der pauschalen Anpassung der Dosierung an das Körpergewicht lässt die unterschiedliche Stoffwechselsituation von Erwachsenen und Kindern vollkommen außer Acht. Die Unterschiede in der Pharmakokinetik bei Erwachsenen und Kindern und hier insbesondere in der Clearance können deutliche Unter- oder Überdosierungen zur Folge haben und machen deutlich, dass "die richtige Dosis so wichtig wie das richtige Medikament" ist.

Auch das Fehlen kindgerechter Darreichungsformen erschwert die adäquate Behandlung und altersgerechte Dosierung insbesondere der sehr kleinen Kinder. Dies führt zur Verabreichung von Arzneimitteln in zerkleinerter und/oder gemörserter Form gemeinsam mit Lebensmitteln. Mögliche Einflüsse solcher Maßnahmen auf die Bioverfügbarkeit der Wirkstoffe sind in den seltensten Fällen untersucht. Aus diesem Dilemma leiten sich konsequenterweise Forderungen nach kindgerechten Formulierungen und Dosierungen $a b$.

Dabei sollten z. B. eine niedrige Applikationsfrequenz, die kindgerechten Applikationsformen einschließlich der Geschmacks- und Geruchsakzeptanz und auch die Entwicklung soziokulturell akzeptabler Darreichungsformen berücksichtigt werden, um die Lebensführung von Kindern und deren Eltern so wenig wie möglich zu beeinträchtigen. Eindeutige, elternverständliche Produktinformationen können die Therapiesicherheit aus Sicht der behandelnden Pädiaterinnen und Pädiater weiter verbessern.
Für die stationäre Behandlungssituation sehen die Kinderärzte die größten Versorgungslücken in der Arzneimittelversorgung im Bereich der Neonatologie und der pädiatrischen Intensivmedizin. In Bezug auf die Fachdisziplinen/ Indikationsgebiete werden Versorgungslücken in den internistischen und neuropädiatrischen/psychiatrischen Bereichen gesehen.

Bereits vor Inkrafttreten der Kinderarzneimittelverordnung (EG) Nr. 1901/ 2006 hatte die pädiatrische Expertengruppe (PEG) der Europäischen Arzneimittelagentur (EMA) in den Jahren 2001-2007 ein Therapiebedarfsinventar aufgestellt. Der PEG gehörten auch Experten/-innen der Kommission für Arzneimittel für Kinder und Jugendliche (KAKJ), die am BfArM eingerichtet ist, an $[7,9]$. Diese Therapieinventarliste wurde durch die Prioritätenliste des Pädiatrieausschusses zu nicht patentgeschützten Wirkstoffen abgelöst, die regelmäßig aktualisiert wird. Analysen dieser Listen [10] zeigen, dass nur ein geringer Teil der PIPs nicht patentgeschützte Wirkstoffe umfasst. Sie unterstützen die Sicht der Kinderärzte, dass in der Vergangenheit zu wenig Gewicht auf die Entwicklung von PUMAZulassungen durch die pharmazeutische Industrie gelegt wurde.

Impulse und Studien aus dem Bereich der nichtkommerziellen Forschung sind von besonderem Interesse für Arzneimittel mit bekannten Wirkstoffen. Gerade für die nichtkommerzielle Forschung sind Förderinstrumente essenziell. In einer vom DLR als Projektträger des BMBF erstellten Analyse zeigte sich, dass sich nur ca. sieben bis acht von 100 eingereichten Projektskizzen der Jahre 2013 bis 2014 auf pädiatrische Fragestellungen bezogen.

Sechs pädiatrische Studien von insgesamt 72 bzw. drei systematische Reviews von insgesamt 102 beantragten Studien wurden mit einem Fördervolumen von rund 7,2 Mio. Euro gefördert.

Aus Sicht des DLR ergeben sich im Vergleich zu anderen klinischen Studien keine grundsätzlich höheren Hürden in der Projektfinanzierung, allerdings erscheint auch hier als größte Herausfor- derung die Rekrutierung geeigneter Patientengruppen [6].

Aus Sicht der Patientenvertretungen wurde noch einmal der Wunsch nach wirksamen und sicheren, d. h. nebenwirkungsarmen Arzneimitteln herausgehoben. Dabei sollte das Augenmerk auch auf eine tatsächliche Verfügbarkeit gelegt werden. Patientinnen und Patienten wünschen sich zeitnahe und praxisorientierte Lösungen auch unter Einbeziehung der Erfahrung von Eltern und Betreuern [5].

\section{Situation aus Sicht der Behör- den}

Eine Vertreterin des BfArM berichtete über den aktuellen Stand der PUMAZulassungen einschließlich der bisherigen Entwicklung [11].

Wie die Europäische Kommission in ihrem aktuellen Bericht vom April 2015 mitgeteilt hatte, wurden alleine in den Jahren 2012 und 201330 neue Arzneimittel mit pädiatrischen Indikationen zugelassen. Weitere 26 patentgeschützte Arzneimittel erhielten im Rahmen von Zulassungsänderungen Indikationen für Kinder und Jugendliche zuerkannt. Im gesamten Zeitraum seit 2007 wurden jedoch nur zwei PUMAZulassungen erteilt. Zuletzt erhielt der B-Adrenorezeptorenblocker Propranolol eine PUMA-Zulassung in der Indikation kindliches Hämangiom. Zuvor war die oro-mukosale Anwendung des Benzodiazepins Midazolam zur Therapie akuter epileptischer Anfälle zugelassen worden.

Eine weitere beantragte PUMAZulassung für ein Influenza-Vakzin (Fluad $^{\circledR}$ ) wurde im Zulassungsverfahren vom Antragsteller zurückgenommen. Lediglich ein weiteres Arzneimittel (Sialanar ${ }^{\circledR}$, Glycopyrronium) befindet sich im Zulassungsverfahren. Die Europäische Kommission nannte dieses Ergebnis in ihrem Fünfjahresbericht 2013 [12] trotz intensiver Fördermaßnahmen enttäuschend. Bei genauerer Betrachtung fällt auf, dass von den 35 in den letzten acht Jahren eingereichten und bewerteten PIPs für bekannte Arzneimittel 22 vom Pädiatrieausschuss positiv bewertet wurden. 13 eingereichte 
Bundesgesundheitsbl 2016·59:1587-1592 DOI 10.1007/s00103-016-2459-8

(c) Der/die Autor(en) 2016. Dieser Artikel ist eine Open-Access-Publikation.

\section{Riedel · B. Lehmann · K. Broich · T. Sudhop}

\section{Arzneimittelzulassung für Kinder und Jugendliche verbessern. Positionspapier zum Kinderarzneimittel-Symposium am 8. Juni 2015 in Bonn}

\section{Zusammenfassung}

Arzneimittel für Kinder und Jugendliche werden in Deutschland und Europa erst seit rund einem Jahrzehnt systematisch so erforscht, wie dies bei Erwachsenen schon seit Jahrzehnten erfolgt. Auch heute werden noch viele Arzneimittel bei Kindern ohne systematische pädiatrische Studien - zum Beispiel zur Dosierung oder zu Darreichungsformen - angewendet. Im Rahmen der EU-Verordnung 1901/2006 sollte geregelt werden, dass Versorgungslücken in der Arzneimitteltherapie für Kinder und Jugendliche geschlossen werden. Dazu sieht die Verordnung für bereits zugelassene und nicht mehr rechtlich geschützte Arzneimittel die Möglichkeit einer Genehmigung für die pädiatrische Verwendung (Paediatric Use Marketing Authorisation - PUMA) vor und räumt den Inhabern dieser PUMA-Zulassung weitergehende Schutzrechte ein. Diese Genehmigung kann für alle pädiatrischen Indikationen in allen oder bestimmten Altersgruppen und für die Entwicklung kindgerechter Darreichungsformen erteilt werden. Die Entwicklung für die Anwendung bei Kindern muss dem durch den Pädiatrieausschuss gebilligten pädiatrischen Prüfkonzept folgen. Dennoch sind seit Inkrafttreten der Verordnung nur zwei PUMA-Zulassungen erfolgreich beantragt worden. Obwohl viele Arzneimittel ein hohes Potenzial aufweisen, große arzneiliche Versorgungslücken für Kinder und Jugendliche zu schließen, wird damit das Instrument der PUMA-Zulassung kaum genutzt.

Zur Verbesserung der Arzneimittelsituation von Kindern und Jugendlichen sollte dieses wissenschaftliche Symposium „More Medicines for Minors" den Dialog unter den beteiligten Partnern verbessern und Gründe für die zurückhaltende Nutzung der PUMAZulassung diskutieren. Vortragende aus dem Bereich der Kinder- und Jugendmedizin, der Zulassungsbehörden, des Gemeinsamen Bundesausschusses, der pharmazeutischen Industrie und der Bundesministerien präsentierten mögliche Probleme und Lösungsmöglichkeiten aus ihrer Sicht mit dem Ziel, das Instrument der PUMA-Zulassungen attraktiver nutzbar zu machen.

Schlüsselwörter

Kinderarzneimittel $\cdot$ Arzneimittelversorgung Arzneimittelzulassung P PUMA · BfArM

\section{Improving drug licensing for children and adolescents. Position paper from the More Medicines for Minors Symposion 8 June 2015 in Bonn}

\begin{abstract}
In Germany and throughout Europe, medicinal products for adults have been developed and evaluated systematically for decades. Medicinal products for children and adolescents, however, have only been researched for the past ten years. As a result, many medicinal products have been administered to children without systematic clinical trials, for example regarding dosage or pharmaceutical form. EU Regulation 1901/2006 aimes to close the gaps in the medical treatment of children and adolescents. In order to do so, the regulation provides for paediatric use marketing authorisations (PUMA) for previously authorised products no longer
\end{abstract}

covered by intellectual property rights and also grants holders of such PUMA licenses further property rights. However, only two PUMA licenses have been applied for. Thus, the PUMA license instrument is hardly being used despite the fact that many medicinal products have a great potential for closing medical gaps for children and adolescents. In order to improve the situation regarding medicinal products for children and adolescents, this scientific symposium "More Medicines for Minors" intended to promote dialogue among the parties involved and to provide an opportunity to discuss reasons for the reluctance to apply for PUMA licenses. Speakers specialised in paediatric and adolescent medicine as well as those from licensing authorities, the Federal Joint Committee (Gemeinsamer Bundesausschuss, G-BA), the pharmaceutical industry and the federal ministries presented problems and possible solutions from their point of view with the aim of making the PUMA license instrument more attractive.

\section{Keywords}

Medicines for children - Medicinal products for the paediatric population - Marketing authorisation for medicinal products . PUMA . BfArM
PIPs wurden durch die antragstellenden Firmen im Verfahren zurückgenommen. Die Gründe, warum bisher nur vier $\mathrm{Zu}$ lassungsanträge letztendlich wirklich eingereicht wurden, sind vielschichtig.

PUMA-Zulassungen benötigen gemäß Artikel 30 der Verordnung (EG) Nr. 1901/2006 einen PIP. Bisher musste dieser PIP die gesamte pädiatrische Population einschließen, d. h. alle Altersklassen umfassen mit der Konsequenz, dass ggf. mehrere Darreichungsformen zu entwickeln wären. Dies war von vielen Antragstellern als zu große Hürde empfunden worden.

Zukünftig akzeptiert die EMA auch PIPs, die nur auf bestimmte pädiatrische Altersklassen begrenzt sind. Darüber hinaus nennt die Europäische Kommission Unsicherheiten und fehlende klare Vorteile in der Erstattung von PUMA-Arzneimitteln als weitere Gründe für die geringe Akzeptanz. Zusammenfassend ist aus Sicht der Europäischen Kommission
- wie auch aus Sicht des BfArM - das Konzept der PUMA-Zulassungen bisher nicht erfolgreich.

Dies führt im Ergebnis auch dazu, dass teilweise die Anwendung des - teureren Kinderarzneimittels umgangen wird, indem der generische Wirkstoff stattdessen auf Verschreibung der Ärzte hin als (kostengünstige) individuell angefertigte Rezeptur aus der Apotheke verwendet wird. Für diese Arzneimittel sind die Maßnahmen zur Pharmakovigilanz wie auch die 
Haftungsregelungen im Vergleich zu zugelassenen Fertigarzneimitteln weniger klar definiert und strukturiert. Insbesondere aus Sicht der Pharmakovigilanz und der Arzneimitteltherapiesicherheit sollten daher zugelassene Fertigarzneimittel (z. B. aus PUMA-Verfahren) für die breitere Anwendung im pädiatrischen Bereich bevorzugt werden.

\section{Situation aus Sicht des Gemeinsamen Bundes- ausschusses (G-BA)}

Anforderungen und damit verbundene Schwierigkeiten bezüglich der Erstattungsfähigkeit sowie mögliche Lösungsansätze wurden durch eine Vertreterin des G-BA erläutert [13].

Obwohl PUMA-Zulassungen mit bekannten Wirkstoffen beantragt werden, fallen sie gemäß $\$ 2$ Abs. 1 der Verfahrensordnung des G-BA unter die Regelungen für Arzneimittel mit neuen Wirkstoffen, da eine PUMA-Zulassung einen Unterlagenschutz gewährt. Damit unterliegen PUMA-Zulassungen in Deutschland bzgl. ihrer Erstattungsfähigkeit ebenfalls der frühen Nutzenbewertung. Als „Nutzen“ sieht die Verfahrensordnung des G-BA patientenrelevante therapeutische Effekte an, so z. B. die Verbesserung des Gesundheitszustands oder der Lebensqualität. Ein Zusatznutzen liegt dann vor, wenn das betreffende Arzneimittel einen qualitativ oder quantitativ höheren Nutzen als die zweckmäßige Vergleichstherapie aufweist. Dabei sind u. a. die Schwere der Erkrankung, die Relevanz und Nachhaltigkeit der beobachteten Effekte sowie andere verfügbare therapeutische Optionen zu berücksichtigen.

Die Anforderungen an die Evidenzlage gemäß Sozialgesetzbuch V orientieren sich an internationalen Standards. Aus Sicht des G-BA können sich Limitationen bzgl. der verfügbaren Evidenz bei PUMA-Zulassungen ergeben.

So sieht die Verordnung (EG) Nr. 1901/ 2006 ausdrücklich zur Verringerung der Patientenzahlen die Nutzung auch von bibliographischen Daten vor, die aus Sicht des G-BA Schwierigkeiten in der vergleichenden Bewertung mit sich bringen können. Der G-BA ist sich der besonderen Herausforderungen in der Durchführung von klinischen Prüfungen mit Kindern bewusst. Neben ethischen Fragestellungen werden vor allem methodische und auch finanzielle Schwierigkeiten bei der Entwicklung von PUMA-Zulassungen gesehen. Sowohl die vermehrte Nutzung von Extrapolationen bei entsprechender medizinischer Rationale wie auch eine vermehrte Einzelfallentscheidung sind entsprechend vom G-BA erwogene Lösungsoptionen.

\section{Situation aus Sicht der pharmazeutischen Industrie}

Vertretende der pharmazeutischen Industrie geben einen Überblick über die Schwierigkeiten bei der Entwicklung und Zulassung von Kinderarzneimitteln [14-17].

Obwohl bei PUMA-Zulassungen der Wirkstoff bereits bekannt und bei Erwachsenen zugelassen ist, ist für PUMAZulassungen im Allgemeinen ein, wenn auch verkürztes, eigenes Entwicklungsprogramm erforderlich.

Dies bezieht sich insbesondere auf die Entwicklung einer angemessenen Darreichungsform mit einer altersgerechten Formulierung und geprüften Dosierungen. Für die Darreichungsform ist zu berücksichtigen, dass nur bestimmte Hilfsstoffe bei Säuglingen und Kleinkindern zulässig sind (Verzicht auf Ethanol, Zucker, Parabene u.a.) und auch die Auswahl geeigneter Geschmacksstoffe verlangt besondere Berücksichtigung. Gerade bei oral verabreichten Arzneimitteln müssen Interaktionen $\mathrm{zu}$ bestimmten Nahrungsmitteln (Milch, Säfte) geprüft werden, ggf. sind auch Dosier- oder Verabreichungshilfen zu entwickeln. Diese Neuentwicklungen im Bereich der pharmazeutischen Formulierung erfordern unter Umständen erneute Bioäquivalenzstudien, die ggf. am Erwachsenen durchgeführt werden können.

Auch bei der Durchführung von Studien an den pädiatrischen Populationen zeigen sich teilweise höhere Schwierigkeiten als bei Erwachsenen. Aufgrund der teilweise deutlich niedrigeren Inzidenz in pädiatrischen Populationen sind mehr Zentren und längere Rekrutierungszeiten erforderlich als bei entsprechenden
Studien bei Erwachsenen, um die notwendigen Fallzahlen zu erzielen $[14,16]$.

Dies erfordert häufig große weltweite Studien mit einer großen Anzahl von Ländern bei kleiner Patientenzahl pro Zentrum. Dies erhöht wiederum aufgrund unterschiedlicher regulatorischer Anforderungen den administrativen Aufwand.

Insgesamt scheint auch die Rekrutierung bei solchen Studien schlechter als bei Erwachsenen zu sein. Grund hierfür ist die geringe Bereitschaft von Eltern, ihre Kinder an klinischen Prüfungen teilnehmen zu lassen. Insbesondere bei nicht vital bedrohlichen Erkrankungen scheint dies häufiger der Fall zu sein [17]. Wegen der teilweise relativen geringen Inzidenzen kommt es bei bestimmten Erkrankungen (z. B. Diabetes, Epilepsie) häufiger zur konkurrierenden Rekrutierung mit anderen Zulassungsstudien, u. a. auch mit innovativen Substanzen. Da hier die Durchführung von PIP-Studien obligat auch für die Zulassung im Erwachsenenbereich ist, treten diese mit freiwilligen PUMA-Zulassungen in Konkurrenz.

Die hohen finanziellen Aufwendungen müssen aus Sicht der pharmazeutischen Industrie finanziell rentabel sein. Entsprechende Erstattungsmöglichkeiten müssen einen wirtschaftlichen Anreiz darstellen. Damit kommt dem Aspekt der Kostenerstattung ein wesentliches Steuerungselement zu. PUMA-Zulassungen unterliegen der frühen Nutzenbewertung. Aus Sicht der Verbände besteht deshalb ein erhebliches Risiko, dass diese Arzneimittel automatisch unter die Festbetragsregelungen fallen, falls der G-BA im Bewertungsverfahren keinen Zusatznutzen für eine PUMA-Zulassung anerkennt. Das bedeutet, dass für diese Arzneimittel nur sehr geringe Erstattungsbeträge vorgesehen sind, vergleichbar mit denen mit generischen Wirkstoffen. Während für z. B. „Orphan Drugs“, also Arzneimittel zur Behandlung seltener Erkrankungen, Regelungen des G-BA automatisch einen Zusatznutzen durch die Zulassung als belegt ansehen, ist dies für PUMAZulassungen bisher nicht der Fall.

Obwohl PUMA-Zulassungen speziell auf pädiatrische Populationen ausge- 
richtet sind, sind sie nicht automatisch vom Austausch in der Apotheke (z. B. im Rahmen der Aut-idem-Regelungen) oder durch den behandelnden Arzt ausgenommen. Damit könnte aus Sicht der Industrie das eigentlich mit der PUMA-Zulassung intendierte Ziel des Unterlagenschutzes umgangen werden.

\section{Diskussion}

Die Diskussion zeigte, dass das jetzige Konzept der PUMA-Zulassung aus Sicht der meisten Vortragenden und Diskussionsteilnehmer/-innen nicht erfolgreich war. Die Gründe liegen zum einen im hohen Aufwand, der für eine PUMAZulassung zu betreiben ist; zum anderen in der Unsicherheit eines finanziellen „return on investment“. Es wurden unterschiedliche Lösungsansätze vorgeschlagen:

Auf europäischer Ebene sind die Vorgaben für die Erstellung eines PIPs limitierend. Die Abschaffung des Erfordernisses, im PIP alle Altersklassen abzudecken, kann den Entwicklungsaufwand bei bestimmten Wirkstoffen deutlich reduzieren und es erlauben, therapiebedarfsgerecht PUMA-Zulassungen zu entwickeln.

Auch die unlängst bekanntgemachte Ausweitung von Freistellungen (Waiver) für bestimmte Altersklassen und Indikationen stellt eine Verfahrensvereinfachung dar ${ }^{2}$.

Aus Sicht der Verbände der pharmazeutischen Industrie stellen vor allem Unsicherheiten bei der Erstattung von PUMA-zugelassenen Arzneimitteln das wesentliche Hindernis dar. Auf nationaler Ebene erscheinen daher besonders Erstattungsregelungen als wesentliches Steuerungs- und Anreizelement. Die Verbände der pharmazeutischen Industrie fordern zudem, PUMA-Zulassungen von den Austauschregelungen

\footnotetext{
2 Mitteilung der Europäischen Kommission: „Leitlinien für Format und Inhalt der Anträge auf Billigung oder Änderung eines pädiatrischen Prüfkonzepts und von Anträgen auf Freistellung oder Zurückstellung sowie für die Überprüfung auf Übereinstimmung und Kriterien für die Bewertung der Signifikanz von Studien" (2008/C 243/01) und ergänzt um die Fassung 2014/C 338/01.
}

(Aut-idem, Substitutionsausschlussliste) grundsätzlich auszunehmen [15].

Da gerade im Bereich der PUMAZulassungen häufig kleinere Studien mit kürzerer Behandlungsdauer und kleineren Fallzahlen notwendig sind, können nichtkommerziell gesponserte Studien hier einen wichtigen Beitrag leisten. Die Problematik der schwierigen Rekrutierung lässt sich durch Netzwerkbildung mildern. Gut funktionierende Netzwerke sind auch für kommerzielle Sponsoren von Interesse, sodass hier Gemeinschaftsprojekte zwischen universitärer Forschung und pharmazeutischer Industrie hilfreich sein können. Eine nachhaltige Förderung bzw. Unterstützung durch öffentliche Mittelgeber ist ebenfalls von großer Bedeutung.

Die nur geringe Tendenz von Eltern, ihre Kinder an klinischen Prüfungen teilnehmen zu lassen, sollte Anlass für aufklärungs- und vertrauensbildende Maßnahmen sein [17]. Auch die verstärkte Einbeziehung von Kindern und Jugendlichen in diese Diskussion könnte eine Änderung in der Haltung der Eltern begünstigen.

Eine 2015 durchgeführte Umfrage der EMA bei Eltern und Kindern zu Kenntnissen und zur Motivation zur Teilnahme an klinischen Prüfungen soll diese Problematik besser beleuchten und dazu beitragen, Lösungsstrategien zu entwickeln [18].

\section{Projektziel}

Die abschließende Diskussionsrunde machte deutlich, dass alle Beteiligten ein hohes Interesse an weiteren PUMAZulassungen haben und die Entwicklung unterstützen möchten. Das BfArM wird daher in Kooperation mit dem Bundesministerium für Gesundheit den Dialog mit allen relevanten Gruppen fortsetzen. Besonders die Therapiebereiche/Indikationen, in denen Versorgungslücken für Kinder und Jugendliche bestehen, sollten eruiert werden.

Als Grundlage einer solchen Liste sollte der Therapiebedarfsinventarplan der EMA dienen, der unter Mitwirkung deutscher Kinderärztinnen und -ärzte erstellt wurde und der die Versorgungslücken in der Pädiatrie aufzeigt [9].
Dieser zeigt, dass insbesondere in den Bereichen kardiovaskuläre Erkrankungen, Endokrinologie, Gastroenterologie, Infektiologie, Neurologie, Onkologie und Ophthalmologie aktuell Versorgungslücken in der Pädiatrie bestehen. Sie umfassen teilweise die fehlende pädiatrische Darreichungsform, aber auch eine nicht nachgewiesene Wirksamkeit und/oder Sicherheit. Zukünftig sollte mit allen verantwortlichen Beteiligten auf eine pädiatrische Zulassung dieser Wirkstoffe hingearbeitet werden.

\section{Korrespondenzadresse}

\section{Dr. C. Riedel}

Bundesinstitut für Arzneimittel und

Medizinprodukte (BfArM)

Kurt-Georg-Kiesinger Allee 3, 53175 Bonn,

Deutschland

claudia.riedel@bfarm.de

\section{Einhaltung ethischer Richtlinien}

Interessenkonflikt. C. Riedel, B. Lehmann, K. Broich und T. Sudhop geben an, dass kein Interessenkonflikt besteht.

Dieser Beitrag beinhaltet keine von den Autoren durchgeführten Studien an Menschen oder Tieren.

Open Access Dieser Artikel wird unter der Creative Commons Namensnennung 4.0 International Lizenz (http://creativecommons.org/licenses/by/4.0/deed. de) veröffentlicht, welche die Nutzung, Vervielfältigung, Bearbeitung, Verbreitung und Wiedergabe in jeglichem Medium und Format erlaubt, sofern Sie den/die ursprünglichen Autor(en) und die Quelle ordnungsgemäßnennen, einen Linkzur Creative Commons Lizenz beifügen und angeben, ob Änderungen vorgenommen wurden.

\section{Literatur}

1. Knöppel C, Klinger O, Soergel M, Seyberth HW Leonhardt A (2000) Anwendung von Medikamenten außerhalb der Zulassung oder ohne Zulassung bei Kindern. Monatsschr Kinderheilkd 148:904-908

2. Bücheler R, Meissner C, Kalchthaler B et al (2002) "Off-label“ Verschreibung von Arzneimitteln in der ambulanten Versorgung von Kindern und Jugendlichen. Dtsch Med Wochenschr 127:2551-2557

3. Mühlbauer B, Janhsen K, Pichler J, Schoettler $P$ (2009) Off-label use of prescription drugs in childhood and adolescence - an analysis of prescription patterns in Germany. Dtsch Arztebl Int 106(3):25-31. doi:10.3238/arztebl.2009.0025 Zugegriffen: 28. April 2016

4. Knopf H, Wolf I-K, Sarganas G, Zhuang W, Rascher W, Neubert A (2013) Off-label medicine use in 
children and adolescents: results of a populationbased study in Germany. BMC Public Health 13:631

5. Bode G, Kinderarzneimittel-Symposium "More Medicines for Minors" (2015) Impulsvortrag über Arzneimittelversorgung aus Sicht der Patientinnen und Patienten. http://www.bfarm. de/SharedDocs/Downloads/DE/Service/Termineund-Veranstaltungen/veranstalt/2015/150608/

2_Bode.pdf Zugegriffen: 28. April 2016

6. Grundmann A, Kinderarzneimittel-Symposium "More Medicines for Minors" (2015) Pädiatrische Studien aus Sicht der Forschungsförderung. http://www.bfarm.de/SharedDocs/Downloads/ DE/Service/Termine-und-Veranstaltungen/ veranstalt/2015/150608/9_Grundmann.pdf Zugegriffen: 28. April 2016

7. Rascher W, Kinderarzneimittel-Symposium „More Medicines for Minors" (2015) Versorgungslücken in der Kinder- und Jugendmedizin schließen - Arzneimittelzulassung mit bewährten Wirkstoffen stärken. http://www.bfarm.de/ SharedDocs/Downloads/DE/Service/Termineund-Veranstaltungen/veranstalt/2015/150608/ 3_Rascher.pdf Zugegriffen: 28. April 2016

8. Zepp F, Kinderarzneimittel-Symposium „More Medicines for Minors" (2015) Versorgungslücken aufdecken aus pädiatrischer Sicht. http://www. bfarm.de/SharedDocs/Downloads/DE/Service/ Termine-und-Veranstaltungen/veranstalt/2015/ 150608/8_Zepp.pdf Zugegriffen:28. April 2016

9. EMA (2015) Needs for paediatric medicines. http:// www.ema.europa.eu/ema/index.jsp?curl=pages/ regulation/document_listing/document_listing 000096.jspZugegriffen:28. April 2016

10. Wimmer S, Rascher W, McCarthy S, Neubert A (2014) The EU paediatric regulation: still a large discrepancy between therapeutic needs and approved paediatric investigation plans. Pediatr Drugs 16(5):397-406

11. Lehmann B, Kinderarzneimittel-Symposium "More Medicines for Minors" (2015) Impulsvortrag über die europäische IST-Situation bei "Paediatric use marketing authorisations" (PUMA-Zulassungen). http://www.bfarm.de/ SharedDocs/Downloads/DE/Service/Termineund-Veranstaltungen/veranstalt/2015/150608/ 1_Lehmann.pdf Zugegriffen:28. April 2016

12. EMA (2013) Better medicines for children from concept to reality. Progress report on the Paediatric Regulation (EC) No 1901/2006. http:// ec.europa.eu/health/files/paediatrics/2013_ com443/paediatric_report-com(2013)443_en. pdf Zugegriffen: 28 . April 2016

13. Löbker W, Kinderarzneimittel-Symposium „More Medicines for Minors" (2015) Besondere Anforderungen an Studien für Kinderarzneimittel (PUMA) aus Sicht der Nutzenbewertung des G-BA. http://www.bfarm.de/ SharedDocs/Downloads/DE/Service/Termineund-Veranstaltungen/veranstalt/2015/150608/ 7 Loebker.pdf Zugegriffen:28. April 2016

14. Bejeuhr G, Kinderarzneimittel-Symposium „More Medicines for Minors" (2015) Hürden für die pharmazeutische Industrie bei PUMA-Zulassungen. http://www.bfarm.de/SharedDocs/Downloads/ DE/Service/Termine-und-Veranstaltungen/ veranstalt/2015/150608/5_Bejeuhr_VFA.pdf Zugegriffen: 28. April 2016

15. Kroth E, Kinderarzneimittel-Symposium „More Medicines for Minors" (2015) Hürden für die pharmazeutische Industrie bei PUMA-Zulassungen. http://www.bfarm.de/SharedDocs/Downloads/ DE/Service/Termine-und-Veranstaltungen/ veranstalt/2015/150608/4 Kroth BAH.pdf Zugegriffen: 28. April 2016

16. Pfeiffer D, Kinderarzneimittel-Symposium „More Medicines for Minors" (2015) Erfahrungsbericht über die erfolgreiche Umsetzung einer PUMA-Zulassung von Propranolol beim kindlichen Hämangiom. http://www.bfarm.de/ SharedDocs/Downloads/DE/Service/Termineund-Veranstaltungen/veranstalt/2015/150608/ 10_Pfeiffer.pdf Zugegriffen:28. April 2016

17. Serrano $P$, Kinderarzneimittel-Symposium „More Medicines for Minors" (2015) Hürden für die pharmazeutische Industrie bei PUMA-Zulassungen. http://www.bfarm.de/SharedDocs/Downloads/ DE/Service/Termine-und-Veranstaltungen/ veranstalt/2015/150608/6_Peters_BPI.pdf Zugegriffen: 28. April 2016

18. EMA (2015) Fragebogen für Kinder und Jugendliche - Meinung in Bezug auf die Anwendung von Medikamenten. http://www.bfarm. de/SharedDocs/Downloads/DE/Arzneimittel/ Zulassung/kam/Fragebogen_EMA-Umfrage.pdf Zugegriffen:28. April 2016 